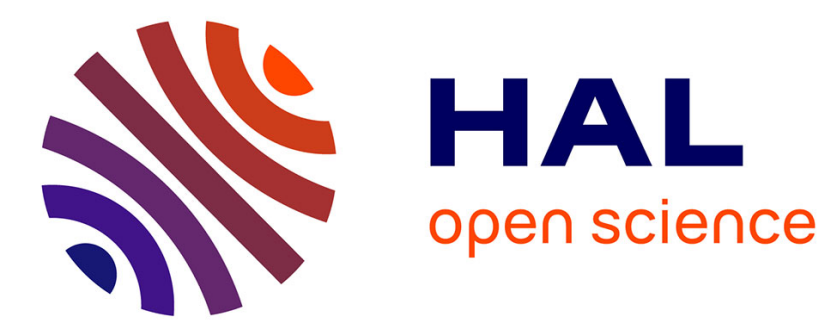

\title{
How a Curved Elastic Strip Opens
}

Thomas Barois, Loïc Tadrist, Catherine Quilliet, Yoël Forterre

\section{To cite this version:}

Thomas Barois, Loïc Tadrist, Catherine Quilliet, Yoël Forterre. How a Curved Elastic Strip Opens. Physical Review Letters, 2014, 113 (21), 10.1103/PhysRevLett.113.214301 . hal-01431992

\section{HAL Id: hal-01431992 https://hal.science/hal-01431992}

Submitted on 11 Jan 2017

HAL is a multi-disciplinary open access archive for the deposit and dissemination of scientific research documents, whether they are published or not. The documents may come from teaching and research institutions in France or abroad, or from public or private research centers.
L'archive ouverte pluridisciplinaire HAL, est destinée au dépôt et à la diffusion de documents scientifiques de niveau recherche, publiés ou non, émanant des établissements d'enseignement et de recherche français ou étrangers, des laboratoires publics ou privés. 


\title{
How a Curved Elastic Strip Opens
}

\author{
Thomas Barois, ${ }^{1,2}$ Loïc Tadrist, ${ }^{1}$ Catherine Quilliet, ${ }^{3}$ and Yoël Forterre, ${ }^{4, *}$ \\ ${ }^{1}$ Department of Mechanics, LadHyX, École Polytechnique-CNRS, 91128 Palaiseau, France \\ ${ }^{2}$ Université Grenoble Alpes, LEGI, F-38000 Grenoble, France CNRS, LEGI, F-38000 Grenoble, France \\ ${ }^{3}$ Université Grenoble Alpes/CNRS UMR 5588, LIPhy, 38041 Grenoble, France \\ ${ }^{4}$ Aix-Marseille Université, CNRS UMR 7343, IUSTI 13453 Marseille Cedex 13, France \\ (Received 3 June 2014; revised manuscript received 7 October 2014; published 21 November 2014)
}

An elastic strip is transversely clamped in a curved frame. The induced curvature decreases as the strip opens and connects to its flat natural shape. Various ribbon profiles are measured and the scaling law for the opening length validates a description where the in-plane stretching gradually relaxes the bending stress. An analytical model of the strip profile is proposed and a quantitative agreement is found with both experiments and simulations of the plates equations. This result provides a unique illustration of smooth nondevelopable solutions in thin sheets.

DOI: 10.1103/PhysRevLett.113.214301

Geometry-induced rigidity is a fundamental feature of thin structures [1], which has long been used in engineering and architecture to design stiff fuselages, hulls, roofs, and deployable structures [2-4]. It is also widely encountered in living structures, such as in plant leaves where curvature can prevent the collapse of the leaves under their own weight [5]. A simple illustration of this rigidity induced by curvature is given by a strip of paper held at one end. When the strip is flat, it is unable to sustain its own weight and bends downward under gravity. However, if the end of the paper is slightly curved transversely, the strip straightens up and becomes much stiffer. Rigidity in these systems arises because bending in one direction is coupled to the transverse curvature and cannot occur without stretching the sheet-a costly mode of deformation in thin plates in terms of elastic energy [6]. Knowing the distance over which an induced curvature spreads is thus an important issue for predicting the rigidity of thin plates and shells.

In this Letter, we address the question of the persistence length of curvature in thin sheets on a minimal system: a flat elastic ribbon of thickness $t$, width $W$, and length $L \gg W$, which is clamped at one end over a cylinder of radius $R$ [Fig. 1(a)]. After what distance from the clamp does the ribbon unfold and recover its flat natural shape? This deceptively simple problem is actually not straightforward as, to unfold, the ribbon has to stretch-a forbidden mode of deformation in the inextensible limit. In thin sheets, this constraint is usually resolved by focusing the stretch in elastic defects or singularities, such as the ridges and peaks of a crumpled paper, the rest of the surface being fully developable (i.e. free of stretching) [7-14]. However, another way to obtain the stretching of a thin sheet is to consider that the curvature variation on large distances is associated with a regular stretching, i.e., without defects. Surprisingly, the first insights of this approach are found in the studies of defects such as ridges [15] and pinches [16],
PACS numbers: 46.32.+x, 46.25.-y, 46.70.-p, 62.20.-x

where both the focused-stress and the diffuse-stress are present [17]. In each of these situations, regular developable solutions exist away from the defect but they are not observed as the bending energy can be progressively released by a small in-plane stretching (see also $[18,19]$ ). The stretching over large distances is also involved in the shape of drapes [20] and curtains [21], the tearing of sheets [22], or the dynamics of curved ribbons [23,24]. Our prototypal system provides a reduced model to probe these situations and the transition between smooth and singular solutions in strained sheets.

A first observation of the opening is displayed in Fig. 1(b), using an acetate elastic sheet $(t=110 \mu \mathrm{m}, W=4.5 \mathrm{~cm})$ clamped in a circular frame of radius $R=2.5 \mathrm{~cm}$. The strip is positioned vertically to limit the out-of-plane deflection caused by its weight. Away from the clamping, the strip opens and its curvature decreases and connects to the flat stress-free region over a finite length $L_{p}$. The opening also results in a small deflection of the strip corresponding to a tilt angle $\theta$ of the centerline. The persistence length of curvature $L_{p}$ can be estimated from a balance between the stretching and bending elastic energies using scaling arguments similar to [15]. On one hand, the bending energy of the ribbon scales as $\mathcal{E}_{b} \sim E t W L_{p}(t / R)^{2}$, where $E$ is the Young modulus of the medium. On the other hand, the opening of the ribbon requires the stretching of the edges of the ribbon over a length $L_{p}$. This is associated with a stretching energy $\mathcal{E}_{s} \sim E t W L_{p} \epsilon^{2}$, where $\epsilon \sim \mathcal{Z}^{2} / 2 L_{p}^{2}$ is the typical in-plane strain and $\mathcal{Z} \sim W^{2} / 8 R$ is the out-of-plane deflection of the ribbon at the clamp [see Fig. 1(b) inset]. The trade off between the two energies $\mathcal{E}_{s}$ and $\mathcal{E}_{b}$ gives $L_{p} \sim W^{2} / \sqrt{t R}$ or equivalently [15] $L_{p} \sim W \sqrt{\mathcal{Z} / t}$. The persistence length of the curved region is independent of the Young modulus and increases when the thickness is reduced. 
(a)

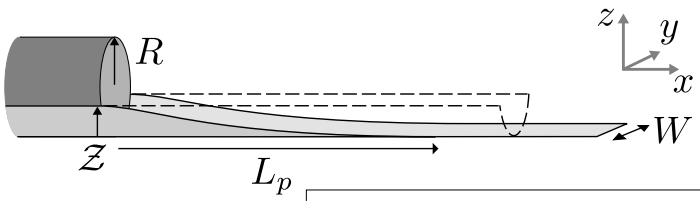

(b)

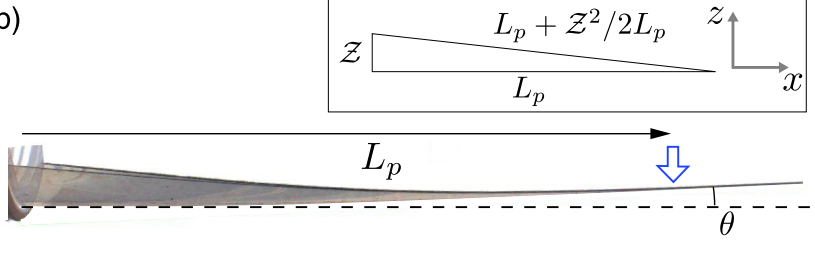

FIG. 1 (color online). (a) Schematic view of a flat strip of width $W$ clamped onto a cylinder of radius $R$ (the dashed lines signal the cylinder surface associated with no in-plane stretching in the strip). $\mathcal{Z}=R[1-\cos (W / 2 R)]$ is the imposed out-of-plane deflection and $L_{p}$ is the opening length. (b) Snapshot of the opening profile obtained with an acetate sheet $(W=4.5 \mathrm{~cm}$, $t=110 \mu \mathrm{m}, R=2.5 \mathrm{~cm}, L_{p}=14.2 \mathrm{~cm}$ ). The arrow signals a deflection comparable to the ribbon thickness. A black background is added to visualize the slight tilt angle $\theta$ of the centerline $y=0$. Inset: the opening of the ribbon over a distance $L_{p}$ requires a stretching of the longitudinal material lines.

To test this scaling law and accurately measure the shape of the ribbon, a modified moiré technique [25] is used, in which a grid pattern made of horizontal lines is projected at low angle on the sheet [Figs. 2(a) and 2(b)]. The deflection of the ribbon in the $z$ direction is obtained from the phase shift of the fringes pattern along $y$ [Fig. 2(c)]. Figure 2(d) shows the curvature profile $c(x)$ along the strip centerline $y=0$, for strips having the same width and initial curvature ( $W=4 \mathrm{~cm}, R=5.5 \mathrm{~cm}$ ) but different thicknesses $t$. The persistence length of curvature increases when the thickness decreases, which implies that the shape of the ribbon involves both stretching and bending modes of deformation. When the distance to the clamp $x$ is normalized by the persistence length scaling $W \sqrt{\mathcal{Z} / t}$, the profiles collapse [Fig. 2(d) left].

Figure 3 shows the measured persistence length $L_{p}$ as a function of $\mathcal{Z} / t$ for the whole range of ribbon thickness, width, and radius of curvature studied experimentally. Within the range of deflection accessible to the experiment ( $\mathcal{Z}$ from $t$ to $100 \times t$ ), the persistence length is in a good agreement with the scaling law proposed before (exponent $1 / 2$ ). This scaling is also confirmed by numerical simulations of the plates equations using Surface Evolver [26]. The low-deflection regime $\mathcal{Z}<t$ is difficult to observe experimentally but can be studied using the numerical simulations. For $\mathcal{Z}<t$, the persistence length seems to be bounded by a lower limit $L_{p} \sim W / 2$. Note that the collapse of the persistence length using the single dimensionless number $\sqrt{\mathcal{Z} / t}$ was not obvious a priori, since dimensional analysis states that the shape of the ribbon might depend also on the wrapping parameter $W / R$. This parameter characterizes the degree of rolling of the ribbon around
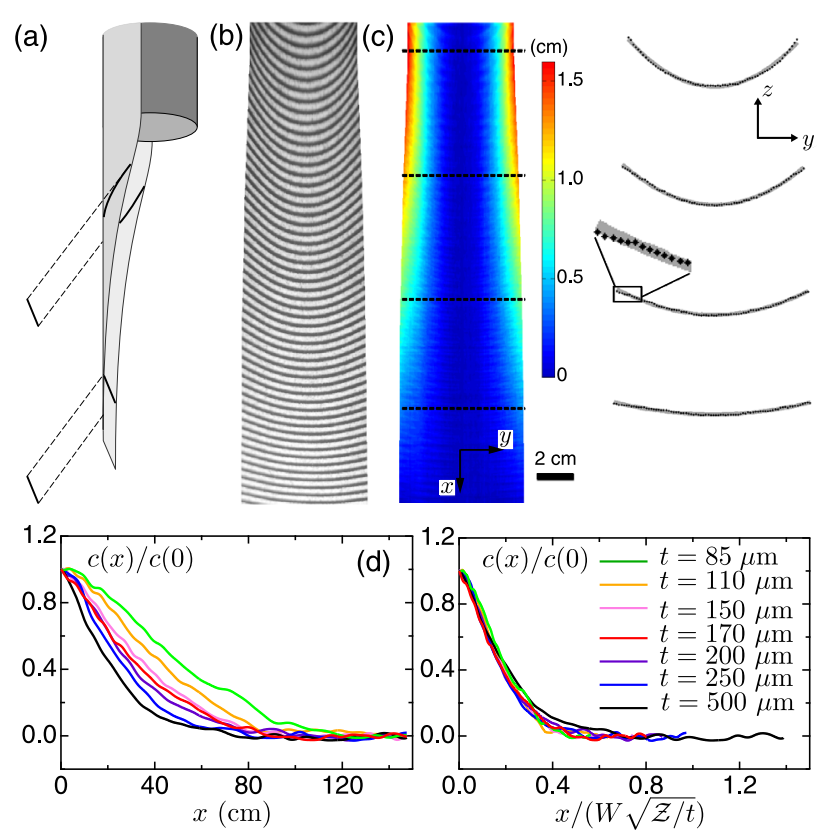

FIG. 2 (color online). (a) Sketch of the modified moiré technique used to measure the ribbon shape (only two fringes are drawn for clarity). (b) Snapshot of the fringes pattern when the ribbon is deflected $(W=7 \mathrm{~cm}, t=76 \mu \mathrm{m}, R=3.5 \mathrm{~cm}$ ), and (c) corresponding ribbon deflection $\mathcal{Z}(x, y)$ relative to the centerline deduced from (b). Four transverse profiles (dashed lines) are selected and compared to parabolic fitting functions (solid lines). (d) Curvature profiles $c(x)$ along the strip centerline $y=0$ for elastic ribbons of different thicknesses $t(W=4 \mathrm{~cm}$, $R=5.5 \mathrm{~cm})$.

the circular clamp as shown in Fig. 4. The numerical simulations confirm that $W / R$ has a weak influence on the persistence length as long as $W \lesssim R$. However, for highly wrapped ribbons $(W / R \sim 2 \pi)$, the persistence length tends to increase [Fig. 4].

The scaling law derived so far only gives the dependence of $L_{p}$ with the dimensions of the ribbon but not the numeric prefactor of the law. In the following, we introduce a 1D model that allows for an analytical and quantitative solution of the strip opening profile. This type of description is possible here because a strip has three separated scales $t \ll W \ll L_{p}$ and a bending deformation imposed to the intermediate scale $W$. For large deflections $\mathcal{Z} \gg t$, the first inequality $t \ll W$ implies that only pure bending is allowed in the transverse direction. Moreover, because of the second inequality $W \ll L_{p}$ (discussed above), the longitudinal bending can be neglected. Consequently, the only possible mechanical coupling is between the transverse bending and the longitudinal stretching. To describe this nonlinear coupling, we use a description inspired by the kinematics of a ruled surface, except that here, the moving line defining the surface can be curved: any strip profile is given by the translation in the $x$ direction of an inextensible curved line with curvature $c(x)$. This description is compatible with the 


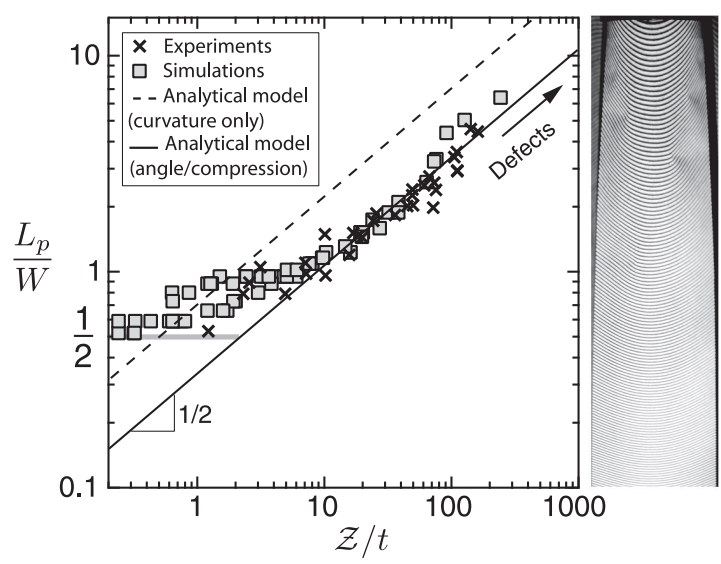

FIG. 3. Normalized persistence length $L_{p} / W$ as a function of the normalized deflection $\mathcal{Z} / t$ for all experimental data (crosses, $W=3-16 \mathrm{~cm}, t=50-2000 \mu \mathrm{m}, R=1.5-5.5 \mathrm{~cm}$ ) and simulations (squares, $0.25<Z / t<243,0.01<W / R<1.5$ ). The persistence length in both cases is defined by $c\left(L_{p}\right)=0.03 \times c(0)$. Dashed line: prediction of the one-dimensional model with curvature only [Eq. (4)]. Solid line: prediction of the model with the compression and the tilt angle [Eq. (8)]. Right insert: picture of the curved ribbon showing a spontaneous nucleation of defects for large deflections $(\mathcal{Z} / t=605)$.

observation of Fig. 2(c) in which the transverse profiles are nearly parabolic. The interest of this approach is to circumvent the coupled equations of plates [30,31] and simplify the computation of curvature energies: the bending energy per unit surface is then simply given by a $1 \mathrm{D}$-function $E t^{3} c(x)^{2} / 24$ [32] in which the Poisson ratio has been set to zero.

The strip opening is first considered for a sheet described by its transverse curvature $c(x)$ only (rigid centerline). The

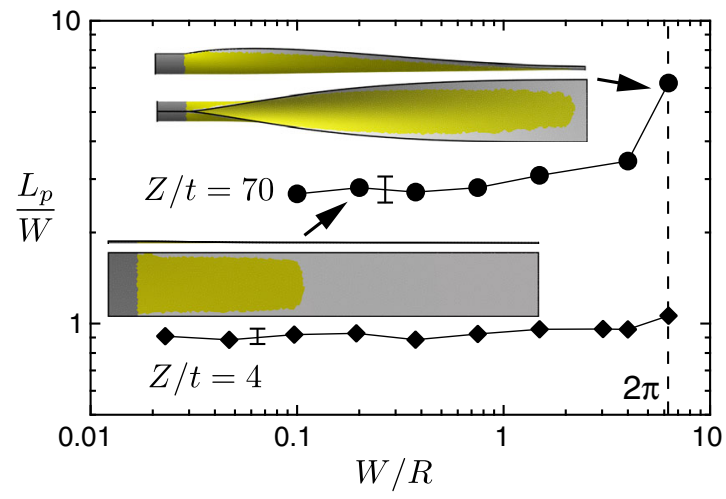

FIG. 4 (color online). Persistence length as function of the wrapping parameter $W / R$ for $\mathcal{Z} / t=4$ (diamonds) and $\mathcal{Z} / t=70$ (bullets) obtained from the numerical simulations. The images show the simulated shape of the curved ribbon (side and top view) for $\mathcal{Z} / t=70$, in the case of a low rolling $(W / R=0.2)$ and a complete rolling around the circular clamp $(W / R=2 \pi)$. The yellow area corresponds to a transverse curvature greater than $3 \%$ of the imposed initial curvature. The error bars give the typical dispersion on several simulations. variation of the transverse curvature $c(x)$ induces a stretching of the longitudinal lines because of the deflection out of plane. When $W / R \ll 1$, the deflection is given by $\mathcal{Z}(x, y)=c(x) y^{2} / 2$. If $d x$ is the distance between two points, this distance is stretched to $d x+\delta d x=$ $d x \sqrt{1+(\partial \mathcal{Z} / \partial x)^{2}}$ and the in-plane displacement is $\varepsilon_{x x}=\delta d x / d x=c^{\prime 2} y^{4} / 8$. This deformation introduces a stretching energy per unit surface given by $E t \varepsilon_{x x}{ }^{2} / 2$. The total elastic energy is given by the sum of the bending and stretching energies:

$$
\mathcal{E}_{0}\left(c, c^{\prime}\right)=\int_{x, y}\left[\frac{1}{2^{3} \times 3} E t^{3} c(x)^{2}+\frac{1}{2^{7}} E t c^{\prime}(x)^{4} v^{8}\right] d x d y,
$$

and after integration on $y \in[-W / 2 ; W / 2]$, the energy is

$$
\mathcal{E}_{0}\left(c, c^{\prime}\right)=\int_{0}^{\infty}\left[\gamma c^{2}+\alpha c^{\prime 4}\right] d x
$$

where $\gamma=E t^{3} W /\left(2^{3} \times 3\right)$ and $\alpha=E t W^{9} /\left(2^{15} \times 3^{2}\right)$. The equilibrium profile for $c(x)$ corresponds to $\delta \mathcal{E} / \delta c=0$ and leads to

$$
12 \alpha c^{\prime \prime} c^{\prime 2}=2 \gamma c
$$

The solution of Eq. (3) that connects to the flat configuration for $x=L_{p}$ is a parabolic profile $c(x)=\left(x-L_{p}\right)^{2} / \lambda^{3}$, with $\lambda^{3}=4 \sqrt{3 \alpha / \gamma}$. The clamping condition is $c(0)=1 / R$ and the persistence length is

$$
L_{p, c}=\frac{1}{4} \frac{W^{2}}{\sqrt{t R}}=W \frac{1}{\sqrt{2}}\left(\frac{\mathcal{Z}}{t}\right)^{1 / 2} .
$$

This first model with $c(x)$ as a unique shape function gives the expected scaling exponent $1 / 2$ (dashed line in Fig. 3), which means that it correctly describes the coupling between bending and stretching deformations. However, the prefactor for the scaling law is not satisfying. This is because, first, the opening has been described with a rigid centerline, which does not agree with the strip profile exhibiting a tilt angle $\theta$ when no vertical force is applied on the strip [see Fig. 1(b)]. Second, in this first model, the longitudinal stretching is always positive $\varepsilon_{x x}=c^{\prime 2} y^{4} / 8>0$, which means that the strip would undergo a net longitudinal mechanical tension, again incompatible with the free end boundary condition [26].

To get an optimal agreement between theory and experiment, one should a priori consider the opening of the strip surface with all the possible displacement of a moving curved line in the 3D space. To do so, two parameters are introduced to allow for the displacement of the centerline in the longitudinal direction and in the out-of-plane direction [ $a_{x}(x)$ and $a_{z}(x)$; note that the reference state is chosen as 
the tilted flat strip far away the clamp, see Fig. 5(a)]. As the location of the edge is $\left[x+a_{x}(x)\right] \vec{x}+\left[a_{z}(x)+\mathcal{Z}(x, y)\right] \vec{z}$, the stretched length of a material line of length $d x$ is now $d x \sqrt{\left(1+a_{x}{ }^{\prime}\right)^{2}+\left[(\partial \mathcal{Z} / \partial x)+a_{z}{ }^{\prime}\right]^{2}}$. At first order, the stretching displacement is modified to $\varepsilon_{x x}=a_{x}{ }^{\prime}+$ $c^{\prime 2} y^{4} / 8+a_{z}{ }^{\prime} c^{\prime} y^{2} / 2$ and the stretching energy now equals

$$
\begin{aligned}
\mathcal{E}_{a_{x}, a_{z}}\left(c, c^{\prime}\right)= & \mathcal{E}_{0}\left(c, c^{\prime}\right)+\int_{0}^{\infty}\left[\tau a_{x}{ }^{\prime 2}+\mu a_{x}{ }^{\prime} c^{\prime 2}+\beta a_{z}{ }^{\prime} c^{\prime 3}\right. \\
& \left.+\delta a_{z}{ }^{\prime 2} c^{\prime 2}+\eta c^{\prime} a_{x}{ }^{\prime} a_{z}{ }^{\prime}\right] d x
\end{aligned}
$$

with $\tau=E t W / 2, \mu=E t W^{5} /\left(2^{7} 5\right), \beta=E t W^{7} /\left(2^{10} 7\right), \delta=$ $E t W^{5} /\left(2^{7} 5\right)$, and $\eta=E t W^{3} /\left(2^{3} 3\right)$. The equilibrium solutions for $a_{x}{ }^{\prime}$ and $a_{z}{ }^{\prime}$ correspond to the functional derivatives $\delta \mathcal{E} / \delta a_{x}{ }^{\prime}=0$ and $\delta \mathcal{E} / \delta a_{z}{ }^{\prime}=0$. After some algebra, these two equations give

$$
a_{x}{ }^{\prime}=-\frac{3}{2^{7} \times 5 \times 7} W^{4} c^{\prime 2} \text { and } a_{z}{ }^{\prime}=\frac{3}{2^{3} \times 7} W^{2} c^{\prime} .
$$

When the expressions for $a_{x}{ }^{\prime}$ and $a_{z}{ }^{\prime}$ are introduced in Eq. (5), the elastic energy simplifies to

$$
\mathcal{E}_{a_{x}, a_{z}}\left(c, c^{\prime}\right)=\int_{0}^{\infty}\left[\gamma c^{2}+\alpha\left(\frac{8}{35}\right)^{2} c^{\prime 4}\right] d x .
$$

The introduction of $a_{x}{ }^{\prime}$ and $a_{z}{ }^{\prime}$ yields a reduction of the stretching term by a factor $(8 / 35)^{2}$. The persistence length is thus changed to

$$
L_{p,\left\{c, a_{x}, a_{z}\right\}}=W \frac{2}{\sqrt{35}}\left(\frac{\mathcal{Z}}{t}\right)^{1 / 2} .
$$

With this expression, the experiments and simulations are remarkably reproduced in Fig. 3 for $10<\mathcal{Z} / t<100$ (solid line). The model is also providing the entire surface profile of the ribbon. Figure 5(a) displays the snapshot of a ribbon opening $(W=3 \mathrm{~cm}, t=110 \mu \mathrm{m}$, and $R=2.5 \mathrm{~cm}$ ) with the superimposition of the profile predicted by the model with the parameters $a_{x}$ and $a_{z}$. Quantitative agreement is obtained not only for the curvature profile [Fig. 5(b)] but also for the tilt angle, as long as $\mathcal{Z} / t$ is large [Fig. 5(c)]. While the deformation of the centerline is small $\left(\left|a_{x}{ }^{\prime}\right|<0.1 \%, a_{z}{ }^{\prime}<0.06\right)$, its influence is significant because the persistence length is reduced by a factor $\sqrt{35 / 8} \approx 2.1$.

In the previous analysis, the curvature energy is only attributed to the energy for the bending of the transverse lines of the strip [transverse bending $c(x)$ ]. The bending in the longitudinal direction might be estimated by $\partial^{2} \mathcal{Z} / \partial x^{2} \sim \mathcal{Z} / L_{p}^{2} \sim t / W^{2}$. The transverse curvature is prescribed by the cylindrical anchor $c \sim 1 / R$. The ratio between these two curvature terms is $R \partial^{2} \mathcal{Z} / \partial x^{2} \sim W^{2} / L_{p}^{2}$. This dimensionless number indicates that the transverse

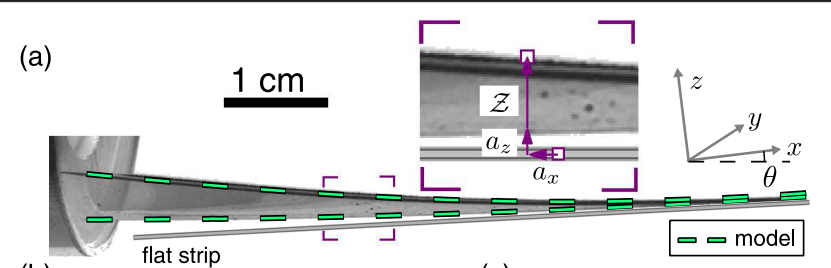

(b)
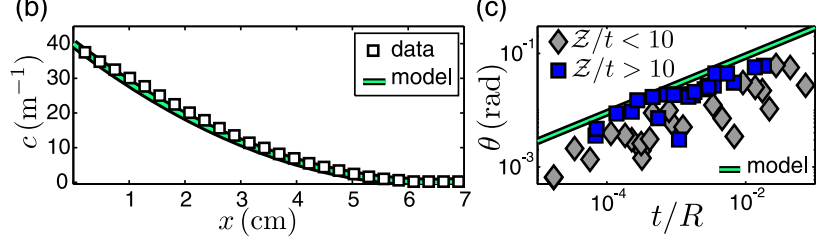

FIG. 5 (color online). (a) Snapshot of a strip clamped in a cylindrical frame of radius $R$ with the superimposition of the profile predicted by the model with the curvature deformations (b). The model uses the solution of Eq. (7), without free parameters. $W=3 \mathrm{~cm}, t=110 \mu \mathrm{m}, R=2.5 \mathrm{~cm}$. (c) Numerical simulation of the total deflection angle as a function of normalized thickness $t / R$ for small $(\mathcal{Z} / t<10)$ and large $(\mathcal{Z} / t>10)$ transverse deflection at the clamp. The solid line is the angle dependence predicted by the model $\left[a_{z}{ }^{\prime}\left(L_{p}\right)-a_{z}{ }^{\prime}(0)\right]$.

curvature and the longitudinal curvature have the same order of magnitude if the persistence length approaches the width of the ribbon. This situation occurs when $\mathcal{Z} \ll t$, i.e. when stretching effects are negligible. The equilibrium profile for the ribbon is then a minimal surface [33] imposing the same curvature $1 / R$ (and the same profile) in the $x$ and $y$ directions. Consequently, for the smallest deflections, the persistence length should equal $W / 2$ [see Fig. 3].

We have demonstrated the existence of smooth diffusestress solutions in thin sheets, where bending and stretching balances over a distance that diverges when the thickness vanishes. The analytical model presented here is simple and quantitative and could be easily adapted to predict the persistence lengths of other geometries. In the case of strips with varying width $W(x)$, the dimensional prefactors in Eqs. (2) and (5) depend on the distance $x$. For precurved strips, the bending energy per unit length equals $E t^{3}\left[c(x)-c_{n}\right]^{2} / 24$, where $c_{n}$ is the natural transverse curvature.

Unlike in [17], the opening of the sheet in our case is not accompanied by a stress-focusing zone at the transition between the curved and flat states. However, for large deflections (typically $\mathcal{Z} / t>430$ in the experiment), this purely smooth solution is no longer observed and pairs of defects spontaneously nucleate close to the frame [see the right insert in Fig. 3]. These defects coexist with the diffuse-stress region as in [17] and are similar to the developable cones observed during the indentation of thin plates $[9,10]$. Understanding the parameters that control this smooth or singular transition is an open issue that should be relevant to many situations involving the confinement of thin sheets. 
We conclude by noting a possible implication of our study in botany for understanding the shape of some plant leaves like maize or grass (monocots familly). During growth, the leaves of these plants unfold and flatten while their base remains attached to the cylindrical stem. Besides genetic factors [34], one may wonder to what extent the open length of these leaves is constrained by mechanics, and the same elastic balance between bending and stretching as the one studied here. An additional ingredient is, however, that leaves' shapes results from a growth (i.e. plastic) process, which should be taken into account to fully address this question.

We acknowledge Basile Audoly, José Bico, Arezki Boudaoud, and Jérémy Hure for fruitful discussions. We also thank K. Brakke for developing and maintaining the Surface Evolver software.

T. B. and L. T. contributed equally to this work.

*Corresponding author. yoel.forterre@univ-amu.fr

[1] B. Audoly and Y. Pomeau, Elasticity and Geometry: From Hair Curls to the Nonlinear Response of Shells (Oxford University Press, Oxford, 2010).

[2] J. E. Gordon, Structures, or Why Things Don't Fall Down, 2nd ed. (Da Capo Press, Cambridge, 1978).

[3] M. Salvadori, Why Buildings Stand Up: The Strength of Architecture (W. W. Norton, New York, 2002).

[4] K. A. Seffen and S. Pellegrino, Proc. R. Soc. A 455, 1003 (1999).

[5] B. Moulia and M. Fournier, J. Mater. Sci. 32, 2771 (1997).

[6] L. Rayleigh, Proc. R. Soc. London 45, 105 (1888).

[7] T. A. Witten, Rev. Mod. Phys. 79, 643 (2007).

[8] M. Ben Amar and Y. Pomeau, Proc. R. Soc. A 453, 729 (1997).

[9] E. Cerda and L. Mahadevan, Phys. Rev. Lett. 80, 2358 (1998).

[10] S. Chaïeb, F. Melo, and J.-C. Géminard, Phys. Rev. Lett. 80, 2354 (1998).

[11] A. Boudaoud, P. Patrício, Y. Couder, and M. Ben Amar, Nature (London) 407, 718 (2000).

[12] A. Vaziri and L. Mahadevan, Proc. Natl. Acad. Sci. U.S.A. 105, 7913 (2008).
[13] A. Lazarus, H. C. B. Florijn, and P. M. Reis, Phys. Rev. Lett. 109, 144301 (2012).

[14] D. Vella, A. Ajdari, A. Vaziri, and A. Boudaoud, Phys. Rev. Lett. 109, 144302 (2012).

[15] A. E. Lobkovsky and T. A. Witten, Phys. Rev. E 55, 1577 (1997).

[16] L. Mahadevan, A.Vaziri, and M. Das, Europhys. Lett. 77, 40003 (2007).

[17] R. D. Schroll, E. Katifori, and B. Davidovitch, Phys. Rev. Lett. 106, 074301 (2011).

[18] P. J. de Pablo, I. A. T. Schaap, F. C. MacKintosh, and C. F. Schmidt, Phys. Rev. Lett. 91, 098101 (2003).

[19] C. D. Santangelo, Soft Matter 9, 8246 (2013).

[20] E. Cerda, L. Mahadevan, and J. M. Pasini, Proc. Natl. Acad. Sci. U.S.A. 101, 1806 (2004).

[21] H. Vandeparre, M. Piñeirua, F. Brau, B. Roman, J. Bico, C. Gay, W. Bao, C. N. Lau, P. M. Reis, and P. Damman, Phys. Rev. Lett. 106, 224301 (2011).

[22] E. Bayart, A. Boudaoud, and M. Adda-Bedia, Phys. Rev. Lett. 106, 194301 (2011).

[23] O. A. E. Arriagada, G. Massiera, and M. Abkarian, Soft Matter 10, 3055 (2014).

[24] F. Guinot, S. Bourgeois, B. Cochelin, and L. Blanchard, Int. J. Solids Struct. 49, 73 (2012).

[25] O. Pouliquen and Y. Forterre, J. Fluid Mech. 453, 133 (2002).

[26] See Supplemental Material at http://link.aps.org/ supplemental/10.1103/PhysRevLett.113.214301, which includes Refs. [1,27-29], for the details of the numerical simulations.

[27] W. Helfrich, Z. Naturforsch. 28c, 693 (1973).

[28] L. D. Landau and E. M. Lifschitz, Theory of Elasticity (Elsevier, New York, 1996).

[29] K. Brakke, Exp. Math. 1, 141 (1992).

[30] A. Föppl, Vorlesungen ü. technische Mechanik, vol. 5 (Leipzig, 1907).

[31] T. von Kármán, Encyk. D. Math. Wiss. IV, 311 (1910).

[32] E. H. Mansfield, The Bending and Stretching of Plates (Cambridge University Press, Cambridge, England, 1989).

[33] P.-G. De Gennes, F. Brochard-Wyart, and D. Quéré, Capillarity and Wetting Phenomena (Springer, New York, 2004).

[34] S. Coen, A.-G. Rolland-Lagand, M. Matthews, J. Bangham, and P. Prusinkiewicz, Proc. Natl. Acad. Sci. U.S.A. 101, 4728 (2004). 